schitz condition of any positive order [3, p. 136], and more general conditions on $f^{(4)}(x)$ are known $[3$, p. 143].

\title{
BiBLIOGRAPHY
} 1947

1. H. S. Carslaw and J. C. Jaeger, Conduction of heat in solids, Oxford, Clarendon,

2. J. Douglas, On the numerical integration of quasi-linear parabolic differential equations, to appear in the Pacific Journal of Mathematics.

3. Antoni Zygmund, Trigonometrical series, Warsaw-Lwow, 1935.

Humble Oil and Refining Company and

DUKE UNIVERSITY.

\section{ON $n$-POINT EXPANSIONS OF ENTIRE FUNCTIONS}

\section{R. C. $\mathrm{BUCK}^{1}$}

In a recent paper [5], A. J. MacIntyre has studied the representation of entire functions of exponential type in certain $n$-point expansions associated with the names Lidstone, Whittaker, Gontcharoff, and Poritsky. In this note, I shall show that the kernel expansion method which I have previously used to discuss general expansion theorems is also applicable to the cases which he has studied.

The general class of expansion formulae that concern us arise from the problem of expressing a function $f$ in terms of the values of a sequence of its derivatives, computed at a finite set of points. Let $\left\{L_{n}\right\}$ be a sequence of functionals defined on the class $K$ of functions of exponential type by

$$
L_{p k+j(f)}=f^{\left(p k+r_{j}\right)}\left(\beta_{j}\right) \quad k=0,1, \cdots ; j=0,1, \cdots, p-1 .
$$

This sequence is specified by the set of integers $\left\{r_{0}, r_{1}, \cdots, r_{p-1}\right\}$ and the set of complex numbers $\left\{\beta_{0}, \beta_{1}, \cdots, \beta_{p-1}\right\}$. The Lidstone set [10] arise from the choice $p=2, r_{0}=r_{1}=0, \beta_{0}=0, \beta_{1}=1$. The generalization introduced by Poritsky [7] corresponds to $r_{j}=0$ for $j=0$, $1, \cdots, p-1$ with arbitrary $\beta_{j}$. The Whittaker two-point set [10] arises when $p=2, r_{0}=0, r_{1}=1, \beta_{0}=1, \beta_{1}=0$, and the periodic Gontcharoff set corresponds to $r_{j}=j$ with arbitrary $\beta_{j}[4]$.

The general problem is to discover to what extent knowledge of the values $L_{n}(f)$ for $n=0,1, \cdots$ determine $f$. If $f$ is of exponential

Received by the editors June 3, 1954.

1 Supported in part by the Office of Ordnance Research. 
type at most $A$, then

$$
f(z)=\frac{1}{2 \pi i} \int_{\Gamma} e^{z w} F(w) d w
$$

where $F$ is the Laplace transform of $f$ and is regular at least for $|w|>A$. The path $\Gamma$ encloses the complete set of singularities of $F$. Using this, the functionals $L_{n}$ have the representation

$$
L_{n}(f)=\frac{1}{2 \pi i} \int_{\Gamma} u_{n}(w) F(w) d w
$$

where

$$
u_{p k+j}(w)=\left(w^{p}\right)^{k} w^{r_{j}} e^{\beta_{j} w} .
$$

To apply the method of kernel expansion in the present case, we seek an expansion of $e^{z w}$ in the form $e^{z w}=\sum_{0}^{\infty} p_{n}(z) u_{n}(w)$ which is uniformly convergent (or summable) for $w$ on $\Gamma$, and for all $z$ in compact sets. Combining this with (2), we have $f(z)=\sum_{0}^{\infty} L_{n}(f) p_{n}(z)$ with $L_{n}(f)$ given by $(3)$.

Since the central point of the present note is to discuss methods rather than detailed results, we treat the representative Lidstone case alone $[1 ; 8 ; 9 ; 10 ; 11]$. We therefore seek to write $e^{z w}$ in the form $\sum_{0}^{\infty} p_{n}(z) w^{2 n}+\sum_{0}^{\infty} q_{n}(z) w^{2 n} e^{w}$. It is easily seen that

$$
e^{z w}=\frac{\sinh (1-z) w}{\sinh w}+e^{w} \frac{\sinh z w}{\sinh w} .
$$

The function $\sinh z w / \sinh w$ is regular for $|w|<\pi$ and is even in $w$ so that it has the power series development $\sum_{0}^{\infty} \Lambda_{n}(z) w^{2 n}$ convergent for $|w|<\pi$, where $\Lambda_{n}$ is a polynomial. Using this, we have $e^{z w}$ $=\sum_{0}^{\infty} \Lambda_{n}(1-z) w^{2 n}+\sum_{0}^{\infty} \Lambda_{n}(z) w^{2 n} e^{w}$, where both series converge for $|w|<\pi$. Proceeding as above (or in [2] or [8]) we conclude that any function $f$ of exponential type $A<\pi$ has the convergent expansion $f(z)=\sum_{0}^{\infty} \Lambda_{n}(1-z) f^{(2 n)}(0)+\sum_{0}^{\infty} \Lambda_{n}(z) f^{(2 n)}(1)$ which is the desired Lidstone series for $f$. As indicated in [2] or in [5], summability may also be discussed. From this Lidstone expansion, we see that if $f$ is of type less than $\pi$ and if $f^{(2 n)}(0)=f^{(2 n)}(1)=0$ for $n=0,1,2, \cdots$, then $f(z) \equiv 0$. Schoenberg [9] using a theorem of Carmichael on difference equations [3] showed that the only functions of exponential type which obey the conditions $f^{(2 n)}(0)=f^{(2 n)}(1)=0$ are the functions $\sin \pi z, \sin 2 \pi z, \cdots$ and their linear combinations. By combining the discussion given above with a device used by W. T. Martin [6] these results may be obtained easily. We observe that the limitation 
$|w|<\pi$ arises from the fact that sinh $w$ which occurs in (5) has simple zeros at $\pm \pi i$. The residue of $\sinh z w / \sinh w$ at $\pm \pi i$ is $\mp i \sin \pi z$ so that

$$
\frac{\sinh z w}{\sinh w}=2 \pi \frac{\sin \pi z}{w^{2}+\pi^{2}}+G_{1}(z, w)
$$

where $G_{1}(z, w)$ is regular for $|w|<2 \pi$ and even in $w$. More generally,

$$
\frac{\sinh z w}{\sinh w}=\pi \sum_{1}^{N} \frac{(-1)^{n+1} n \sin (n \pi z)}{w^{2}+n^{2} \pi^{2}}+G_{N}(z, w)
$$

where $G_{N}(z, w)=\sum_{0}^{\infty} g_{n}(z) w^{2 n}$, convergent for $|w|<(N+1) \pi$. The function $g_{n}(z)$ is the sum of the polynomial $\Lambda_{n}(z)$ and a finite trigonometric sum. This in turn gives

$$
\begin{aligned}
e^{z w}= & \sum_{0}^{\infty} g_{n}(z) w^{2 n} e^{w}+\sum_{0}^{\infty} g_{n}(1-z) w^{2 n} \\
& +\pi \sum_{1}^{N} \frac{n \sin (n \pi z)}{w^{2}+n^{2} \pi^{2}}\left[1+(-1)^{n+1} e^{w}\right]
\end{aligned}
$$

convergent for $|w|<(N+1) \pi$ and we obtain in the same way as before the following result.

Theorem. If $f$ is of exponential type $A<(N+1) \pi$ then

$$
f(z)=\sum_{0}^{\infty} g_{n}(1-z) f^{(2 n)}(0)+\sum_{0}^{\infty} g_{n}(z) f^{(2 n)}(1)+\sum_{1}^{N} C_{n} \sin (n \pi z)
$$

where

$$
C_{n}=\frac{n}{2 i} \int_{\Gamma} \frac{1+(-1)^{n+1} e^{w}}{w^{2}+n^{2} \pi^{2}} F(w) d w
$$

and $\Gamma$ may be taken as the circle $|w|=\rho$ with $A<\rho<(N+1) \pi$.

Corollary. If $f$ is of exponential type $A<(N+1) \pi$ and $f^{(2 n)}(0)$ $=f^{(2 n)}(1)=0$ for $n=0,1,2, \cdots$ then $f(z)=\sum_{1}^{N} C_{n} \sin (n \pi z)$.

It is clear that the same approach may be used to discuss the other cases treated by MacIntyre [5] as well as the more general case studied incompletely by Schmidli [8] and that it has many advantages over that used by the former.

\section{REFERENCES}

1. R. P. Boas, Representation of functions by Lidstone series, Duke Math. J. vol. 10 (1943) pp. 239-245. 
2. R. C. Buck, Interpolation series, Trans. Amer. Math. Soc. vol. 64 (1948) pp. 283-298.

3. R. D. Carmichael, Summation of functions of a complex variable, Ann. of Math. vol. 33 (1936) pp. 349-378.

4. W. Gontcharoff, Détermination des fonctions entières par interpolation, Paris, 1937.

5. A. J. MacIntyre, Interpolation series for integral functions of exponential type, Trans. Amer. Math. Soc. vol. 76 (1954) pp. 1-13.

6. W. T. Martin, On expansions in terms of a certain general class of functions, Amer. J. Math. vol. 58 (1936) pp. 407-420.

7. H. Poritsky, On certain polynomial and other approximations to analytic functions, Trans. Amer. Math. Soc. vol. 34 (1932) pp. 274-331.

8. S. Schmidli, Über gewisse Interpolationreihen, Zurich thesis, 1942.

9. I. J. Schoenberg, On certain two-point expansions of integral functions of exponential type, Bull. Amer. Math. Soc. vol. 42 (1936) pp. 284-288.

10. J. M. Whittaker, On Lidstone's series and two point expansions of analytic functions, Proc. London Math. Soc. vol. 36 (1933-1934) pp. 451-469.

11. D. V. Widder, Completely convex functions and Lidstone series, Trans. Amer. Math. Soc. vol. 51 (1942) pp. 387-398.

UNIVERSITY OF WISCONSIN 